\title{
The Laboratory Right Dimension in Drug Design and Efficacy Pharmacological Molecule Modify Strategy- New Algorithm
}

\author{
Luisetto $\mathrm{M}^{1 *}$, Behzad $\mathrm{NA}^{2}$ and Ghulam $\mathbf{R M}^{3}$ \\ ${ }^{1}$ Department Applied pharmacologist, Italy \\ ${ }^{2}$ Department Nano Drug Delivery, USA \\ ${ }^{3}$ Department Medical \& Health Sciences, Peoples University of Medical \& Health Sciences for Woman, Pakistan \\ *Corresponding author: Luisetto M, Applied pharmacologist, European specialist in lab medicine, hospital pharmacist manager, Italy

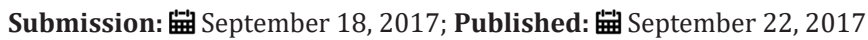

\section{Abstract}

Keywords: Industrial pharmacy; Medicinal chemistry; Drug design; Clinical pharmacy; Clinical pharmaceutical care; Clinical outcomes

\section{Introduction}

As we can easily see a high amount of pharmacological molecules was discovered in the last century and especially during 1900-1970 periods. Then from 1970 to 2017 we can see that few new class of drugs are introduced and a lot of me too pharmacological molecules are been discovered. The same we can see that the legislative and normative involved in drug registration was deeply introduced after Second World War (we can think for example starting from ethical principle HELSINKY; but many other rules was introduced in pharmaceutical field). This condition; in our opinion ; has created a situation in which the development of new drug classes become more difficult under situation of high level of regulation as request today.

We can see also that modify of old and new molecule in order to improve ADME profile or in drug delivery; or other kind of medicinal chemistry molecule modify is currently used to create new drugs or to improve drugs already in commerce. What we can see that it seems that as the development of regulatory rules was associated to a reduced in discovering new drug classes. And this also observing the dimension of the actual pharmaceutical industries and producers.

We can say that the ideal standard to discover and introduce new class in pharmacological molecules

Was the lab research dimension we have seen in 1850-1970 and the related normative rules profile.

In this period the discovering of the new drugs was due in many cases to pure discovery or to the coincidence and not a standardized industrial process. We can think that the right dimension to discover new pharmacological strategy is not a large pharmaceutical industry but it can be single level researcher or laboratory. The creativity in this field can be the right instrument to generate high amount of new idea and project or to treat the problem in an unconventional way. I many example some errors have make possible to introduce very efficacy drugs (FLEMING experience and many others).

The same we can see that some drugs was introduced for example by dentistry (first gas anesthetics) and not from pharmaceutical industries. Other examples can be blood compatibility in which many errors in first application were done before introducing this essential procedure. (Other examples the discovery of pacemaker's medical devices was due by an error but many other examples we can see). The some first antidepressant drugs were first introduced as antimicrobials; sildenafil discovered searching a strategy for angina pectoris and so on. The big pharma have the high power of industries but perhaps the better idea can come from other dimension laboratories. According the editorial "The Clinical Pharmacist Competence as Pharmaceutical Drug Design Tool" 2017 "The clinical pharmacist specific competences added to medicinal chemistry knowledge and clinical need are efficacy instruments to translate to pharmaceutical industries the modification of pharmacological molecule or the need to search new strategy in drug therapy if not efficacy as request" [1].

We can see that adding the clinical pharmacist's competences to the pharmaceutical and medical competencies to the researcher 
we can have more results then without this specific competency [2]. The same the medicinal chemist's competences can add the right strategy to translate the medical and clinician request in produce high efficacy molecules.

\section{Discussion \& Conclusion}

Today we need to rethink the actual model to discover new pharmacological molecules and reintroducing the methods used in the period 1850-1970 to have new really efficacy drug classes. The clinical pharmacists competences added to the specific medicinal chemistry knowledge applied also in small research lab can give more clinical results to be translated to the industrial pharmacy. In this creativity added to the chemistry competences and the knowhow of the clinical pharmacist and the other clinicians can give right strategy to produce drugs whit really efficacy and not only me too drugs [3]. The new algorithm can be in the first step of discovering process not to go on in registration of not really efficacy pharmacological molecules only due by economic purpose but only related to the clinical outcomes goal.

The same after registration process the drugs re-design to improve the clinical efficacy must require a low burocracy as possible. Respecting the ethical principle and the patient safety we think that the creativity added to the single level of researcher activity must not to be compressed by a too much burocratic systems or to a business affairs systems too much oriented to the economic results.

\section{References}

1. Luisetto M, Nili AB (2017) The Clinical Pharmacist Competence as Pharmaceutical Drug Design Tool. RRJHCP 3(2): 1-2.

2. Luisetto M, Nili AB, Ghulam RM (2017) The Clinical Pharmacists Main Focus.

3. M Luisetto (2016) Editorial Efficacy of Oncologic Drug Therapy: Some to Re think in the Management of the System? Journal of Business Management and Economics 4(7): 16-20. 